(1)

CrossMark

\title{
IL-6 in pulmonary hypertension: why novel is not always best
}

\author{
Mark Toshner $\mathbb{1}^{1,2}$ and Alexander M.K. Rothman $\mathbb{1}^{3,4}$ \\ Affiliations: ${ }^{1}$ Royal Papworth Hospital, Cambridge, UK. ${ }^{2}$ Dept of Medicine, University of Cambridge, \\ Cambridge, UK. ${ }^{3}$ University of Sheffield, Sheffield, UK. ${ }^{4}$ Sheffield Teaching Hospitals NHS Trust, Sheffield, UK. \\ Correspondence: Mark Toshner, University of Cambridge, Dept of Medicine, Level 5 Addenbrooke's Hospital, \\ Cambridge CB2 OQQ, UK. E-mail: mrt34amedschl.cam.ac.uk
}

@ERSpublications

Previous preclinical data on IL-6 validated in a large multicentre biobank study. IL-6 is associated with mortality and levels vary related to underlying aetiology. This has relevance to experimental medicine attempts to translate immunomodulatory therapy. https://bit.ly/2WTFUMQ

Cite this article as: Toshner M, Rothman AMK. IL-6 in pulmonary hypertension: why novel is not always best. Eur Respir J 2020; 55: 2000314 [https://doi.org/10.1183/13993003.00314-2020].

In this issue of the European Respiratory Journal, SIMPSON et al. [1] present data from the US National Institutes of Health and National Heart, Lung, and Blood Institute pulmonary arterial hypertension (PAH) biobank on interleukin (IL)-6 levels in a large cohort of patients with pulmonary hypertension. This work is of significant importance, as it potentially represents a new capability in rare cardiorespiratory diseases for large scale multicentre validation studies, rather than the current focus on target and pathway novelty in underpowered, small scale studies that are usually single centre. SIMPSON et al. [1] show, in an unrivalled dataset, that there are differences in serum levels of IL- 6 between PAH aetiologies and differences in the relationship between expression levels and outcomes. Reassuringly, they validate in a large multicentre cohort the core previous observations of increased IL-6. They note differences between disease aetiologies, and in particular that levels are highest in connective tissue disease (CTD)-associated PAH and, perhaps less expectedly, portopulmonary hypertension. The study also can be viewed as resolving the question of whether IL-6 is associated with outcomes. The authors clearly show this to be the case in powerful multivariate modelling accounting for a variety of functional, prognostic and mortality outcomes.

The first demonstration of increased IL-6 dates back 25 years [2] and, since then, multiple groups have confirmed this observation in small cohorts with the additional suggestion of possible associations with long-term outcomes [3, 4]. In murine models, overexpression of IL-6 leads to the development of pulmonary vascular lesions [5], IL-6 deficient mice are protected [6] and administration of an IL-6R neutralising antibody attenuates the development of experimental PAH [7]. Looking wider than IL-6 signalling, there is broad acceptance that inflammation plays an integral part in PAH pathobiology $[8,9]$. The wealth of preclinical data provided a solid platform from which the clinical tractability of inflammation in patients with PAH was to be explored. The repositioned drug, tocilizumab, provided an excellent opportunity with an acceptable side-effect profile, extensive post-market data and limited experience in patient groups directly relevant to $\mathrm{PAH}$, such as scleroderma and rheumatoid arthritis. Although powered to detect a large effect size, a phase 2 study of tocilizumab in PAH (TRANSFORM-UK), demonstrated target engagement, altering C-reactive protein (CRP) and IL-6, with no overall change in pulmonary vascular resistance (PVR) or secondary efficacy parameters [10]. The only positive signal, which should be very cautiously interpreted in a small population, was in prespecified analyses by PAH subgroup, which found that patients with CTD may have had a modest effect on PVR. In $\mathrm{PAH}$, with the exception of targeting proliferation with imatinib [11], no new hypothesis has survived 
through to phase 3 of drug development in the last 20 years. There are of course many reasons drugs fail. In rare disease, however, one obvious problem is the small sample size of early experimental studies and, therefore, it is critical when approaching rare populations to be confident that the best patient cohort for the pathobiological hypothesis has been defined.

Phase 2 and 3 studies of PAH-targeted therapies often enrol patients with a range of underlying aetiologies, creating a diversity within the study population that may influence study outcomes [12]. The single largest population in clinical trials is traditionally idiopathic $\mathrm{PAH}$, where the only major pathobiological advance in our understanding of aetiology in the last 10 years has arguably been the addition of novel rare variants underlying disease, such as the recent demonstrations of mutations in ATP13A3, AQP1 and SOX17 [13]. Large scale multi-national collaborative genetic studies enrolling well-characterised patients with idiopathic PAH appear likely to only lead to the eventual reclassification of less than $5-10 \%$ of additional idiopathic patients as heritable or spontaneous de novo mutation-associated. For the remaining majority of patients, significant further effort will be required before we can be clear what is causing disease. This heterogeneity will impact on the effectiveness of some treatment hypotheses and inflammation is a good potential example of this.

The demonstration by SIMPSON et al. [1] of significant increases in IL-6 levels in CTD-PAH may now be viewed through the prism of the results of TRANSFORM-UK, where this group was the only putative positive signal. Because they were able to assay a large population, we also have more data in less severe disease groups. A previous concern was that IL-6 may simply be increased in end-stage illness or heart failure. It is possible that increased IL-6 is an epiphenomenon of studying severely ill patients. In the current work there are many more patients with modest or mild disease and though there remain significant associations between IL-6 levels and classical parameters of disease progression, in multivariate analysis taking into account these associations there still remains a significant relationship with outcomes. The ability to run multivariate analyses is of importance. The lack of dependence or a relationship to classical haemodynamic parameters suggests that IL-6 signalling is potentially not coupled to either PVR or heart failure, despite the association with poor outcomes. The demonstration here that IL-6 concentrations were increased in pulmonary artery smooth muscle cells (PASMCs), but not endothelial cells, confirms previous work suggesting PASMCs may be a source of increased IL-6 [14]. Whether local vascular and autocrine production of IL-6 is more important than inflammatory cells is an unanswered question but the repeated demonstration of PASMC does at the very least suggest a mechanism whereby IL-6 signalling locally could be uncoupled from classical mediators of mechanical resistance and heart failure. The authors point out that the uncoupling of PVR from IL-6 may mean that PVR is not the ideal study endpoint to use in evaluating the efficacy of IL-6 targeted therapies. If, however, we are to put patients at the additional risks associated with immunomodulation, it is difficult to imagine this being easily justified without a clearly demonstrable effect on vascular resistance and cardiac function. The effect size we should be aiming for with this approach is open for debate.

SIMPSON et al. [1] add significant evidence to the inflammatory hypothesis of PAH. The TRANSFORM-UK trial has, for the moment, demonstrated the challenges translating the inflammatory signalling in patients with $\mathrm{PAH}$, and as such should be viewed as a test of target engagement with an available drug in a study with a diverse patient population rather than a definitive last word on the inflammatory hypothesis. Recent studies of the inflammatory targeting agents canakinumab [15] and colchicine [16] have demonstrated improved clinical endpoints in patients with atherosclerosis and have required very large numbers. It is important to note that CANTOS enrolled only patents with raised inflammatory markers and following treatment with canakinumab a greater clinical benefit was apparent in patients achieving a high-sensitivity CRP (hs-CRP) $<2 \mathrm{mg} \mathrm{L}^{-1}$ [17]. As such, stratification with biomarkers, endotyping or phenotyping is also likely to be necessary in PAH. It should also not be lost that the first randomised clinical study to demonstrate the therapeutic tractability of inflammatory signalling in patients with coronary disease showed a reduction in hs-CRP with therapy; however, hsCRP was increased following drug withdrawal and major adverse cardiac events significantly increased at 1 year [18, 19]. Although without regulatory approval, the persistent examination of multiple therapies targeting related inflammatory pathways eventually demonstrated improvement in hard outcomes.

As we move beyond vasodilation in PAH it may be that there will not be universally applicable class effects from novel treatments and, specifically, this may be the case in inflammation. In vasodilation itself this can also be argued as true, given the failure of translation to groups 2 and 3 pulmonary hypertension. We will need to carefully consider how to examine therapeutic effect in early phase studies and how to stratify populations. By focusing on an important, established, pathobiological hypothesis in a large population rather than pursuing novelty, SIMPSON et al. [1] have given us additional food for thought in how we conduct and run later stage preclinical studies in inflammation and, more generally, in PAH. 
Conflict of interest: M. Toshner reports personal fees from GSK and Morphogen IX, grants and personal fees from J\&J/ Actelion, grants from Merck, Bayer and Roche, outside the submitted work. A.M.K. Rothman reports grants and personal fees from Sonivie, Novartis, Medtronic, Endotronix, Abbott and Actelion, outside the submitted work.

Support statement: M. Toshner has received funding from the Cambridge NIHR BRC, British Heart Foundation and MRC. A.M.K. Rothman is supported by a Wellcome Trust Clinical Research Career Development Fellowship (206632/Z/ 17/Z). Funding information for this article has been deposited with the Crossref Funder Registry.

\section{References}

1 Simpson CE, Chen JY, Damico RL, et al. Cellular sources of interleukin-6 and associations with clinical phenotypes and outcomes in pulmonary arterial hypertension. Eur Respir J 2020; 55: 1901761.

2 Humbert M, Monti G, Brenot F, et al. Increased interleukin-1 and interleukin-6 serum concentrations in severe primary pulmonary hypertension. Am J Respir Crit Care Med 1995; 151: 1628-1631.

3 Soon E, Holmes AM, Treacy CM, et al. Elevated levels of inflammatory cytokines predict survival in idiopathic and familial pulmonary arterial hypertension. Circulation 2010; 122: 920-927.

4 Heresi GA, Aytekin M, Hammel JP, et al. Plasma interleukin-6 adds prognostic information in pulmonary arterial hypertension. Eur Respir J 2014; 43: 912-914.

5 Steiner MK, Syrkina OL, Kolliputi N, et al. Interleukin-6 overexpression induces pulmonary hypertension. Circ Res 2009; 104: 236-244.

6 Savale L, Tu L, Rideau D, et al. Impact of interleukin-6 on hypoxia-induced pulmonary hypertension and lung inflammation in mice. Respir Res 2009; $10: 6$.

7 Tamura Y, Phan C, Tu L, et al. Ectopic upregulation of membrane-bound IL6R drives vascular remodeling in pulmonary arterial hypertension. J Clin Invest 2018; 128: 1956-1970.

8 Sweatt AJ, Hedlin HK, Balasubramanian V, et al. Discovery of distinct immune phenotypes using machine learning in pulmonary arterial hypertension. Circ Res 2019; 124: 904-919.

9 Humbert M, Guignabert C, Bonnet S, et al. Pathology and pathobiology of pulmonary hypertension: state of the art and research perspectives. Eur Respir J 2019; 53: 1801887.

10 Toshner ACC M, Harlow L, Coghlan G, et al. Transform-UK: a phase 2 trial of tocilizumab in pulmonary arterial hypertension. Am J Respir Crit Care Med 2018; 197: A7804.

11 Ghofrani HA, Morrell NW, Hoeper MM, et al. Imatinib in pulmonary arterial hypertension patients with inadequate response to established therapy. Am J Respir Crit Care Med 2010; 182: 1171-1177.

12 Olschewski H, Simonneau G, Galie N, et al. Inhaled iloprost for severe pulmonary hypertension. $N$ Engl J Med 2002; 347: 322-329.

13 Graf S, Haimel M, Bleda $\mathrm{M}$, et al. Identification of rare sequence variation underlying heritable pulmonary arterial hypertension. Nat Commun 2018; 9: 1416.

14 Davies RJ, Holmes AM, Deighton J, et al. BMP type II receptor deficiency confers resistance to growth inhibition by TGF-beta in pulmonary artery smooth muscle cells: role of proinflammatory cytokines. Am J Physiol Lung Cell Mol Physiol 2012; 302: L604-L615.

15 Ridker PM, Everett BM, Thuren T, et al. Antiinflammatory therapy with canakinumab for atherosclerotic disease. N Engl J Med 2017; 377: 1119-1131.

16 Tardif JC, Kouz S, Waters DD, et al. Efficacy and safety of low-dose colchicine after myocardial infarction. $N$ Engl J Med 2019; 381: 2497-2505.

17 Ridker PM, MacFadyen JG, Everett BM, et al. Relationship of C-reactive protein reduction to cardiovascular event reduction following treatment with canakinumab: a secondary analysis from the CANTOS randomised controlled trial. Lancet 2018; 391: 319-328.

18 Morton AC, Rothman AM, Greenwood JP, et al. The effect of interleukin-1 receptor antagonist therapy on markers of inflammation in non-ST elevation acute coronary syndromes: the MRC-ILA Heart Study. Eur Heart J 2015; 36: 377-384.

19 Rothman AM, Morton AC. Crossman DC, et al. Canakinumab for atherosclerotic disease. N Engl J Med 2018; 378: $197-198$. 